\title{
Primary thrombosis of the anterior descending in a patient with antiphospholipid antibody syndrome
}

\author{
Jorge Acuña-Valerio', Marco Antonio Peña-Duque ${ }^{2}$ and Álvaro Contreras-Villaseñor ${ }^{2}$ \\ ${ }^{1}$ Dental and Health Sciences Program, Universidad Nacional Autónoma de México, Based in Instituto Nacional de Cardiología Ignacio Chávez, \\ Mexico; ${ }^{2}$ Department of Hemodynamics, Instituto Nacional de Cardiología Ignacio Chávez, Ciudad de México, Mexico
}

\begin{abstract}
Antiphospholipid antibody syndrome is a heterogeneous clinical and biochemical condition. We present the case of a young male with a history of venous thromboembolism. This time he presents due to chest ischemic pain associated with ST-segment elevation. He was taken to the cath lab to perform a primary percutaneous coronary intervention, and a total occlusion of the left anterior descending artery was noted. Successful thrombus aspiration was performed. No stent was deployed. He was taken to the cath lab for a second look angiography, and no atherosclerotic lesions were observed, which was confirmed by intravascular ultrasound.
\end{abstract}

KEY WORDS: Antiphospholipid antibody syndrome. Antiphospholipid antibodies. Coronary thrombosis. Acute myocardial infarction. Non-reflow phenomenon.

\section{Introduction}

Antiphospholipid antibody syndrome (APS), or Hughes syndrome, is a heterogeneous situation that refers to: (1) presence of antibodies against phospholipids or against phospholipid-binding proteins, which are known as antiphospholipid antibodies (APLA), among which the best characterized are lupus anticoagulant, anticardiolipin, and anti-b2-glycoprotein; (2) at least two clinical manifestations including arterial or venous thrombosis, miscarriage, and thrombocytopenia $^{1,2}$. The criteria to classify patients were published in 1990 and revised in $2006^{3}$.

It can occur in association with other autoimmune disorders (especially systemic lupus erythematosus), which is known as secondary APS, but in up to $50 \%$ of cases, it occurs as a single clinical syndrome, which is classified as primary APS. Other less common form is catastrophic APS ${ }^{4}$.
APS the most common cardiovascular manifestations are arterial or venous thromboses. However, accelerated atherosclerosis syndrome, verrucous endocarditis, cardiac valvular disorders, intracardiac thrombosis with or without cardioembolism, stent thrombosis, pericarditis with or without myocardial involvement, venous bridges' premature degeneration, pulmonary hypertension, arterial hypertension secondary to renal stenosis, and peripheral artery disease have also been associated ${ }^{5}$. Cardiovascular manifestations vary according to whether it is primary APS or associated with other autoimmune disorders ${ }^{6}$.

\section{Case presentation}

This is the case of a 27-year-old male with a history of left iliofemoral deep vein thrombosis (unprovoked) 4 years prior, with subsequent pulmonary thromboembolism. This condition was not associated with any

\section{Correspondence:}

Jorge Acuña-Valerio

Medical Sciences MD \& PhD Program

Universidad Nacional Autónoma de México

Based in Instituto Nacional de Cardiología

Ignacio Chávez Mexico

Date of modified version reception: 09-06-2016

E-mail: drjacuval@gmail.com

Date of acceptance: 10-06-2016

DOI: 10.24875/GMM.M17000036
Gac Med Mex. 2017;153:481-484

Contents available at PubMed www.gacetamedicademexico.com 
clinical risk factor for thromboembolism, and APLA detection was positive. He was treated with conventional heparin and later with a coumarin anticoagulant, and an inferior vena cava filter was placed. He remained asymptomatic and on anticoagulant treatment.

On this occasion, he presented with oppressive precordial pain of sudden onset when walking, which prompted him to attend the hospital. ST-segment elevation was observed on electrocardiogram anterior wall leads. The patient was administered $300 \mathrm{mg}$ of acetylsalicylic acid and $300 \mathrm{mg}$ of clopidogrel and referred to a center with the capacity to perform primary angioplasty. He arrived to our hospital with ischemia time of $3 \mathrm{~h}$. He was observed with tachycardia (110 bpm), tachypnea (26 breaths/minute), and diaphoresis, with no signs of acute heart failure or any other significant cardiopulmonary alteration. A new electrocardiogram showed ST elevation at V1-5, DI, and $\mathrm{aVL}$. He was administered morphine, oxygen, other $300 \mathrm{mg}$ of clopidogrel, and $4000 \mathrm{IU}$ intravenous heparin (his last international normalized ratio (INR) had been 1.51 week prior), and was brought to the hemodynamics room. The radial approach was selected.

The coronarography revealed total ostial occlusion of the anterior descending artery (grade 5 thrombus) (Fig. $1 A$ and $B$ ). No other coronary alterations were detected. A run through floppy guide wire $\left(\right.$ Terumo $^{\circledR}$ ) was advanced, which crossed the obstruction with no difficulty, and a Diver $\left(\right.$ Invatec $\left.^{\circledR}\right)$ manual aspiration system was then advanced, by means of which several fragments of thrombotic material were extracted and vessel patency was restored (Fig. $2 \mathrm{~A}$ and B). Door-to-balloon time was $74 \mathrm{~min}$. Thrombus aspiration was repeated two more times, and a double dose of intracoronary tirofiban was administered. Mild non-reflow phenomenon was observed and adenosine was used, by means of which is improved. No atherosclerosis plaque was identified in subsequent angiographies at different projections, residual thrombus was minimal and flow was almost normal (TIMI 3 MBG 2), and not deploying a stent was therefore decided. Heparin and tirofiban peripheral infusions were continued. Resolution criteria were observed (complete disappearance of pain and ST-segment elevation normalization). $8 \mathrm{~h}$ later, the patient had melena, and an upper GI tract endoscopy was therefore performed, which revealed stress-associated diffuse gastropathy. An echocardiogram revealed left ventricle ejection fraction (LVEF) of $34 \%$, moderate anterior and apical hypokinesis and mild diastolic dysfunction; no valvular or pericardial alterations were identified, and pulmonary pressure was estimated at $42 \mathrm{mmHg}$. An X-ray showed signs of pulmonary congestion. Troponin I peak value was $>150 \mathrm{ng} / \mathrm{mL}$. Vasodilators and diuretics were indicated, with favorable evolution and no need for the use of inotropics.

At $48 \mathrm{~h}$, the patient was brought again to the cath lab, where he underwent left coronarography. The residual thrombus image had disappeared. No coronary lesions were observed. An intracoronary ultrasound probe $\left(\right.$ Volcano $\left.^{\circledR}\right)$ was advanced, with no atherosclerotic plaques being found in the ultrasonographic path along the anterior descending artery or left main coronary artery. As a decrease in the right femoral pulse had been detected since admission, a right iliofemoral angiography was performed, which documented $a<5 \mathrm{~cm}$ occlusion of external iliac and common femoral artery, with subsequent rechanneling and adequate collateral circulation. Since the patient was asymptomatic and the obstruction was not acute, not performing any intervention at that moment was decided.

The patient did not experience precordial pain again or show electrographic changes suggestive of ischemia or new enzyme elevations. Neither did he experience another abnormal hemorrhage. On focused history taking, no family history of early coronary disease or any other cardiovascular risk factor, as well as symptoms suggestive of immune disease were found. The patient was evaluated by the rheumatology specialist, with no alterations suggestive of systemic lupus erythematosus or any other autoimmune disorder being found. Antinuclear antibodies and rheumatoid factor were negative, anticardiolipin IgM was 8.4 MPL/mL (normal $<7 \mathrm{MPL} / \mathrm{mL}$ ), anticardiolipin IgG was > $120 \mathrm{GPL} / \mathrm{mL}$ (normal < $10 \mathrm{GPL}$ ) $\mathrm{mL}$ ), anti- $\beta 2$-glycoprotein IgM was $2.6 \mathrm{U} / \mathrm{mL}$ (normal $<8 \mathrm{U} / \mathrm{mL}$ ), anti-b2-glycoprotein IgG was > $100 \mathrm{U} / \mathrm{mL}$ (normal $<8 \mathrm{U} / \mathrm{mL}$ ), and lupus anticoagulant was positive. Based on the patient history and on this biochemical pattern, and with the precedent of a screening for APLA that was positive, he was diagnosed with primary APS. Before hospital discharge, a new echocardiogram was practiced, which demonstrated ventricular function improvement (LVEF of $45 \%$ with mild anteroapical hypokinesis). He was discharged with statin, acetylsalicylic acid, acenocoumarin, metoproIol, and enalapril, with an INR of 3.0 at 8 days of the coronary event, with an appointment to the outpatient clinic at 6 weeks to define the best peripheral revascularization strategy. 

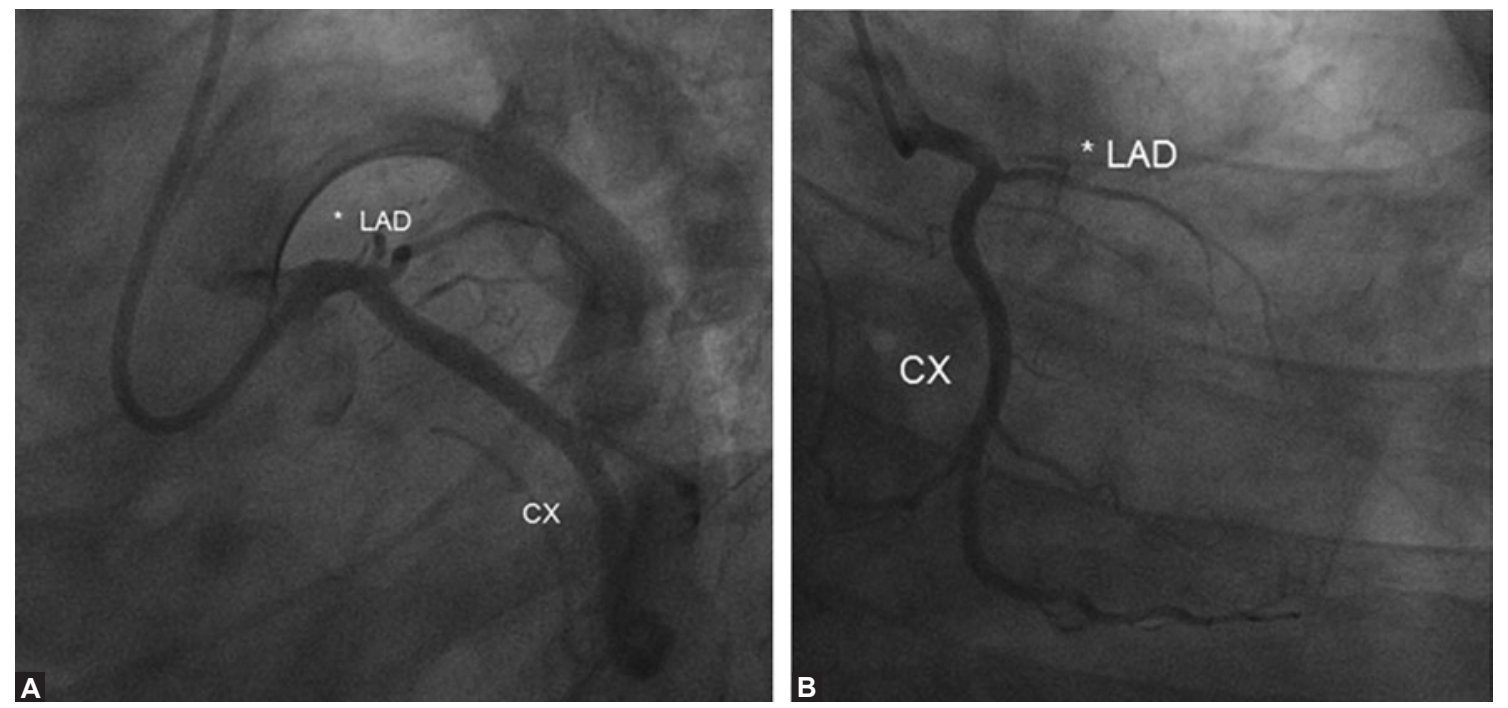

Figure 1. (A and B) Anterior descending artery total occlusion from its ostium. Circumflex artery with no lesions. Right coronary artery, which also had no lesions, is not shown. CX: circumflex artery; LAD: left anterior descending artery.
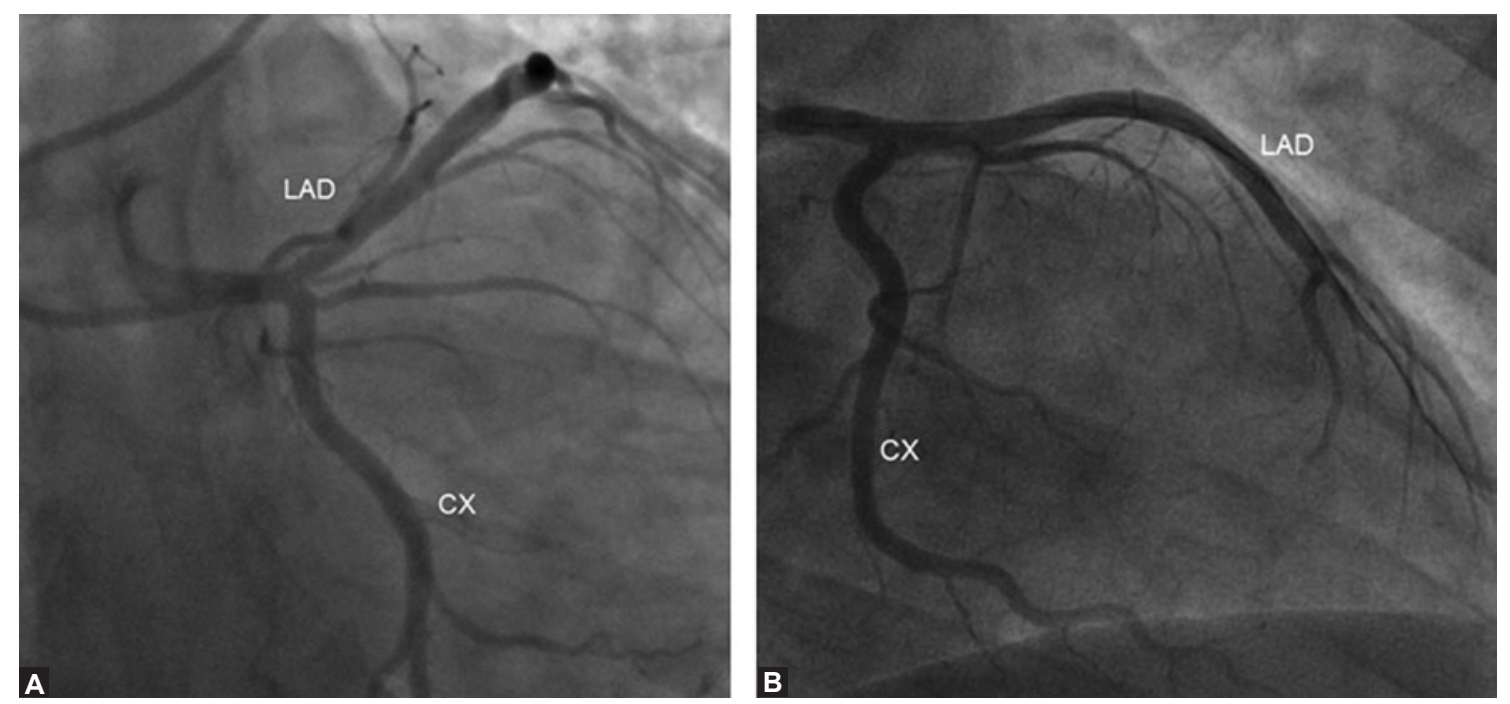

Figure 2. (A and B) After manual aspiration, the anterior descending artery recovered the flow, with no tightening suggestive of atherosclerosis being observed. There is a small laminar thrombus in the ostium (without embolism phenomena at distal branches). In the arteriography practiced 2 days later, the thrombus had completely disappeared. CX: circumflex artery; LAD: left anterior descending artery.

\section{Discussion}

We present the case of a 27-year-old male with a history of pulmonary thromboembolism, who had spent several years asymptomatic on anticoagulant therapy, but this time he presented with acute coronary syndrome due to an anterior descending artery primary thrombosis. No atherosclerotic lesions were found by angiography or intravascular ultrasound, and stents or balloons were therefore not used for interventional treatment. After an episode of acute heart failure, he evolved satisfactorily and was therefore discharged with acetylsalicylic acid and coumarin anticoagulant (INR of 3.0), with peripheral arterial obstruction treatment being left pending.

Although other pathologies, in addition to coronary artery disease, should be considered in a patient with APS presenting with precordial pain, especially if it coexists with lupus or other autoimmune disorders ${ }^{1}$, the presence of pain and electrocardiographic changes suggestive of infarction mandates the clinician to treat the patient as any other subject with suspicion of acute coronary syndrome, regardless of the history of pulmonary thromboembolism, or the use of anticoagulants. 
Acute myocardial infarction is rare as APS initial manifestation. In one study, it accounted for $2.8 \%$ of incident cases, but during follow-up, it was developed by $5.5 \%$ of patients ${ }^{4}$. Coronary thrombosis is estimated to occur in $5 \%$ of people who have APLA detected ${ }^{2}$, and a higher prevalence of APLA has also been observed in patients who suffer acute coronary syndromes with regard to those with stable chronic angina ${ }^{5}$. Only cerebral arterial circulation is more commonly involved than coronary artery circulation in patients with APS (cerebral infarctions, transient ischemia attacks, and dementia of vascular origin) ${ }^{2}$. Coronary obstruction atypical mechanisms have been described $^{6}$. In this patient, alterations were found in two arterial territories, together with a history of venous thromboembolism, with no neurological alterations found on focused history and physical.

In addition, chronic coronary disease is more common in subjects with APS than in the general population. In one study, myocardial changes suggestive of ischemia were observed in $29.6 \%$ of patients with APS (asymptomatic with regard to heart issues), which is comparable to the incidence of coronary artery disease observed in subjects with diabetes or end-stage kidney disease ${ }^{7}$. In this case, no coronary lesions were identified by angiography in any vessel, and their absence was corroborated by means of intracoronary ultrasound at the proximal and medial segments of the anterior descending and left main coronary artery. Magnetic resonance-detected myocardial necrosis is associated rather with APS time of evolution, presence of livedo reticularis, and anti- $\beta 2$-glycoprotein antibodies positive status than with traditional cardiovascular risk factors ${ }^{7}$.

Anticardiolipin antibodies and their cofactor $\beta 2$-glycoprotein I have been associated with incipient atherosclerosis $^{5}$. In addition to the known favorable effects of statins in subjects with acute coronary syndromes, anti- $\beta 2$-glycoprotein-mediated endothelial activation has been described to be able to be inhibited by statins ${ }^{5}$ and, therefore, even in the absence of coronary plaque, we decided to indicate atorvastatin to this patient, whom we consider at high risk for the development of cardiovascular events on the long-term, even when he has not any of the classic coronary risks.

In these individuals, life-long coagulation is mandatory. An INR of 3.0-3.5 is recommended, which is a higher goal than for other hypercoagulation states ${ }^{2}$. Thrombotic events can recur even with INR at therapeutic range $\mathrm{e}^{6,8}$.
The syndrome can coexist with genetic alterations such as Leiden factor $\mathrm{V}$ or other prothrombotic states ${ }^{9}$. Even resistance to clopidogrel has been described in these patients ${ }^{10}$. Therefore, if thrombotic episodes recur in spite of adequate coagulation, other conditions should be suspected.

Peripheral artery disease has also been described in APS, and it can have different presentations ${ }^{11,12}$. In this case, deferring the treatment of this condition until the patient recovered from the coronary event was decided. Although the lesion morphology favored the endovascular treatment option, the topography oriented more toward a surgical approach.

\section{Conclusion}

Due to APS broad clinical spectrum, any clinical manifestation in a patient with this diagnosis should alert the clinician about a possible arterial or venous thrombotic event or else disease reactivation by another mechanism. In this case, by aspiration of the thrombus, total acute thrombotic occlusion of the anterior descending artery could be solved without the need for angioplasty with balloon or stent, since thrombosis was not associated with atherosclerotic plaque.

\section{References}

1. Miranda CH, Gali LG, Marin-Neto JA, et al. Coronary thrombosis as the first complication of antiphospholipid syndrome. Arq Bras Cardiol. 2012;98:e66-9.

2. Asherson RA, Cervera R. Antiphospholipid antibodies and the heart; lessons and pitfalls for the cardiologist. Circulation. 1991;84:920-3.

3. Miyakis S, Lockshin MD, Atsumi T, et al. International consensus statement on an update of the classification criteria for definite antiphospholipid syndrome (APS). J Thromb Haemost. 2006;4:295-306.

4. Cervera R, Piette J, Font J, et al. Antiphospholipid syndrome: Clinical and immunologic manifestations and patterns of disease expression in a cohort of 1,000 patients. Arthritis Rheum. 2002;46:1019-27.

5. Long BR, Leya $F$. The role of antiphospholipid syndrome in cardiovascular disease. Hematol Oncol Clin N Am. 2008;22:79-94.

6. Vianna JL, Khamashta M, Ordi-Ros J, et al. Comparison of the primary and secondary antiphospholipid syndrome: A European multicenter study of 114 patients. Am J Med. 1994;96:3-9.

7. Sacré K, Brihaye B, Hyafil F, et al. Asymptomatic myocardial ischemic disease in antiphospholipid syndrome. Arthritis Rheum. 2010;62: 2093-100.

8. Monteiro AV, Branco LM, de Sousa L, et al. Antiphospholipid syndrome: a recurrent cardiac thromboembolic insult in spite of optimal anticoagulation. Reumatol Clin. 2015;11:177-8.

9. Santos JL, Cruz I, Herrero FM, et al. Trombosis coronaria recurrente, síndrome antifosfolipídico primario, factor $\mathrm{V}$ Leiden y virus de la inmunodeficiencia humana. Rev Esp Cardiol. 2004;57:997-9.

10. Middlebrooks EH, Panda M. Multiple recurrent stent thrombosis in a patient with coexisting clopidogrel resistance and increased anticardiolipin antibodies: a case report. Case Rep Med. 2010;2010:1-5.

11. Toffon A, Piovesan R, Minniti CF, et al. Lower limb ischemia: aortoiliac thrombosis related to antiphospholipid syndrome (APS) - case report and review of literature. Case Rep Surg. 2013;2013:536971.

12. Namazi MH, Kaheshi I, Serati AR, et al. Resistant hypertension due to unilateral renal artery occlusion as the first presentation of antiphospholipid syndrome. Cardiovasc Revasc Med. 2015;16:190-1. 